\title{
Research on brand valuation models based on triangular fuzzy matrix game
}

\author{
Qi Duan', Bi Song Liu², Fang Wu ${ }^{3}$ \\ 1,2, ${ }^{3}$ Quality Management Branch, China National Institute of Standardization, Beijing, 100191, China \\ ${ }^{1}$ School of Economics and Management, Tsinghua University, Beijing, 100084, China \\ ${ }^{3}$ Corresponding author \\ E-mail: ${ }^{1}$ duanqi@cnis.gov.cn, ${ }^{2}$ liubs@cnis.gov.cn, ${ }^{3}$ wufang@cnis.gov
}

Received 31 July 2017; accepted 1 August 2017

DOI https://doi.org/10.21595/vp.2017.18927

Check for updates

\begin{abstract}
Market competition has entered into the era of brand competition with economic growth. Brand evaluation represents an important approach for brand management and value enhancement. In this paper, an analysis was made, based on the triangular fuzzy matrix game theory, on the profits and losses incurred by participating enterprises and evaluating institutions due to their adoption of different game strategies. The applications of triangular fuzzy matrix game theory were expanded to the brand valuation field, and a theoretical method for evaluating the current evaluation models was proposed.
\end{abstract}

Keywords: brand value, brand evaluation, triangular fuzzy, game.

\section{Introduction}

A great brand can help the enterprise sell products, seize the market and reduce the risk of consumers when purchasing products. Thus, Enterprises regard brand management as an important guarantee and way out for their business management and development. As an important part of brand management and a significant indicator for strategic performance of brand, brand valuation can not only effectively reflect the market status of a brand but also provide a reference for the future development policy-making by the enterprise and guide consumers to make purchase in a rational manner. However, the limited cognition of mankind and incomplete information during the brand valuation may impact on the accuracy of the profits and losses predicted and judged by the participating enterprise and the evaluating institution in advance. Thus, in this paper, the triangular fuzzy numbers were used to indicate the profits and losses that are difficult to be expressed when the parties adopted different strategies. The applications of triangular fuzzy matrix game theory were expanded to the brand valuation field. A new method for evaluating the current evaluation models was proposed. Also, evaluation cases were explained.

\section{Triangular fuzzy matrix game}

\subsection{Triangular fuzzy number}

In reality, it is usually difficult for us to make an accurate adjustment when we are faced with an uncertain future or there is no way for us to get clear of the future profits. In such case, we can only get an estimated interval. Nevertheless, the proposing of the triangular fuzzy number improves the accuracy, completeness and practicality of the interval estimation.

Definition 1. Suppose $\tilde{a}=(o, p, q)$ is a triangular fuzzy number and its affiliated function is $\mu_{\tilde{a}}(x): R \rightarrow[0,1]$, namely:

$$
\mu_{\tilde{a}}(x)= \begin{cases}\frac{x}{p-o}-\frac{o}{p-o}, & x \in[o, p], \\ \frac{q}{q-p}-\frac{x}{q-p}, & x \in[p, q], \\ 0, \text { others. } & \end{cases}
$$


Definition 2 . For any two triangular fuzzy numbers $\tilde{a}_{1}=\left(o_{1}, p_{1}, q_{1}\right)$ and $\tilde{a}_{2}=\left(o_{2}, p_{2}, q_{2}\right)$, the possibility degree of $\tilde{a}_{1}>\tilde{a}_{2}$ is:

$V\left(\tilde{a}_{1} \geq \tilde{a}_{2}\right)=\left\{\begin{array}{l}1, \quad p_{1}>p_{2}, \\ \frac{q_{1}-o_{2}}{\left(p_{2}-p_{1}\right)+\left(q_{2}-o_{2}\right)}, \quad p_{1}<p_{2}, \quad q_{1}>o_{2}, \\ 0, \quad q_{1}<o_{2} .\end{array}\right.$

Definition 3. For any $\mathrm{n}$ triangular fuzzy numbers $\tilde{a}_{1}, \tilde{a}_{2}, \ldots, \tilde{a}_{n}$, the possibility degree of a triangular fuzzy number $\tilde{a}_{i} \geq \tilde{a}_{1}, \ldots, \tilde{a}_{n}$ is:

$V\left(\tilde{a}_{i} \geq \tilde{a}_{1}, \ldots, \tilde{a}_{n}\right)=\min \left\{V\left(\tilde{a}_{i} \geq \tilde{a}_{1}\right), V\left(\tilde{a}_{i} \geq \tilde{a}_{2}\right), \ldots, V\left(\tilde{a}_{i} \geq \tilde{a}_{n}\right)\right\}, \quad 1 \leq i \leq n$.

\subsection{Triangular fuzzy matrix game}

In brand valuation, uncertain evaluation result may lead to the adoption of many fuzzy strategies by both game participants. Thus, a triangular fuzzy number may be used to indicate the profits and losses of the parties. And the profits and losses of game participants can be marked as follows:

$\tilde{G}=\left\{S_{1}, S_{2} ; \tilde{A}\right\}$,

wherein, $S_{1}=\left\{\alpha_{1}, \alpha_{2}, \ldots, \alpha_{m}\right\}$ means strategy set of game participant $1 ; S_{2}=\left\{\beta_{1}, \beta_{2}, \ldots, \beta_{n}\right\}$ means strategy set of game participant $2 ; \tilde{A}=\left[\left\{\tilde{a}_{i j}, \tilde{b}_{i j}\right\}\right]_{m \times n}(1 \leq i \leq m, 1 \leq j \leq n)$ is a profit and loss matrix of game participant. When strategy combination is $\left\{\alpha_{i}, \beta_{j}\right\}$, the profit and loss matrix of game participant 1 is $\tilde{a}_{i j}=\left(a_{i j}^{o}, a_{i j}^{p}, a_{i j}^{q}\right)$ and that of game participant 2 is $\tilde{b}_{i j}=\left(b_{i j}^{o}, b_{i j}^{p}, b_{i j}^{q}\right)$.

Definition 4. According to Definition 2, if strategy $\alpha_{i}^{*}, \beta_{j}^{*}$ constitutes the strategy combination $\left\{\alpha_{i}^{*}, \beta_{j}^{*}\right\}$, which meets the condition of $V_{i}^{* *}>0$, the strategy combination $\left\{\alpha_{i}^{*}, \beta_{j}^{*}\right\}$ will be the optimal pure strategy solution based on possibility degree $V_{i}^{* *}$.

Wherein:

$V_{i}^{* *}=V\left(\tilde{a}_{i j}^{* *} \geq \tilde{a}_{i j}^{*}, 1 \leq i \leq n\right) \cdot V\left(\tilde{b}_{i j}^{*^{*}} \geq \tilde{b}_{i j}^{*}, 1 \leq j \leq m\right)$.

According to the above definitions and parameters, the mixed strategies of the participating enterprise and the evaluating institution are:

$X_{m}=\left\{x=\left(x_{1}, x_{2}, \ldots, x_{m}\right) \mid x_{i} \geq 0,1 \leq i \leq m, \quad \sum_{i=1}^{m} x_{i}=1\right\}$,
$Y_{n}=\left\{y=\left(y_{1}, y_{2}, \ldots, y_{m}\right) \mid y_{j} \geq 0, \quad 1 \leq j \leq n, \quad \sum_{j=1}^{n} y_{j}=1\right\}$.

Suppose that the expected profit of the participating enterprise and the evaluating institution is $E(x, y)$.

Assume the profit matrix of the game participants is expressed in Table 1.

Then, the expected profit of the game participant 1 is:

$E_{\text {Enterprise }}(\tilde{x}, \tilde{y})=\tilde{x} \tilde{y} \tilde{a}_{11}+\tilde{x}(1-\tilde{y}) \tilde{a}_{12}+(1-\tilde{x}) \tilde{y} \tilde{a}_{21}+(1-\tilde{x})(1-\tilde{y}) \tilde{a}_{22}$. 
Table 1. Profit matrix of game participants

\begin{tabular}{|c|l|l|l|}
\hline \multirow{4}{*}{ Participant 1} & \multicolumn{3}{|c|}{ Participant } \\
\cline { 2 - 4 } & & $\beta_{1}(\tilde{y})$ & $\beta_{2}(1-\tilde{y})$ \\
\cline { 2 - 4 } & $\alpha_{1}(\tilde{x})$ & $\tilde{a}_{11}, \tilde{b}_{11}$ & $\tilde{a}_{12}, \tilde{b}_{12}$ \\
\cline { 2 - 4 } & $\alpha_{2}(1-\tilde{x})$ & $\tilde{a}_{21}, \tilde{b}_{21}$ & $\tilde{a}_{22}, \tilde{b}_{22}$ \\
\hline
\end{tabular}

Let:

$\frac{\partial E_{\text {Enterprise }}(\tilde{x}, \tilde{y})}{\partial \tilde{x}}=\tilde{y} \tilde{a}_{11}+(1-\tilde{y}) \tilde{a}_{12}-\tilde{y} \tilde{a}_{21}-(1-\tilde{y}) \tilde{a}_{22}=0$.

Then:

$\tilde{y}^{*}=\frac{\tilde{a}_{22}-\tilde{a}_{12}}{\tilde{a}_{11}-\tilde{a}_{12}-\tilde{a}_{21}+\tilde{a}_{22}}$.

That is, in case of optimal mixed strategy, the evaluating institution selects strategy $\beta_{1}$ by using the probability $\tilde{y}^{*}$ and $\beta_{1}$ by using the probability $\beta_{2}$.

Similarly, the expected profit of the game participant 2 is:

$E_{\text {Institution }}(\tilde{x}, \tilde{y})=\tilde{x} \tilde{y} \tilde{b}_{11}+\tilde{x}(1-\tilde{y}) \tilde{b}_{12}+(1-\tilde{x}) \tilde{y} \tilde{b}_{21}+(1-\tilde{x})(1-\tilde{y}) \tilde{b}_{22}$.

Let:

$\frac{\partial E_{\text {Institution }}(\tilde{x}, \tilde{y})}{\partial \tilde{y}}=\tilde{x} \tilde{b}_{11}-\tilde{x} \tilde{b}_{12}+(1-\tilde{x}) \tilde{b}_{21}-(1-\tilde{x}) \tilde{b}_{22}=0$.

Then:

$\tilde{x}^{*}=\frac{\tilde{b}_{22}-\tilde{b}_{12}}{\tilde{b}_{11}-\tilde{b}_{12}-\tilde{b}_{21}+\tilde{b}_{22}}$.

Thus, the optimal mixed strategy solution is $\left(\tilde{x}^{*}, \tilde{y}^{*}\right)$. The meanings of the conclusion can be interpreted as follows (Assume $V\left(\tilde{a}_{11}-\tilde{a}_{12}-\tilde{a}_{21}+\tilde{a}_{22}>0\right)=1$ and $\left.V\left(\tilde{b}_{11}-\tilde{b}_{12}-\tilde{b}_{21}+\tilde{b}_{22}>0\right)=1\right)$.

\section{Brand valuation models}

Brand valuation models may be divided into two types: independent evaluation by the institution and the evaluation at the request of enterprise. The strategy sets of the participating enterprise and the evaluating institution under both types of models are \{participate, not participate\}, \{evaluation at the request of enterprise, independent evaluation by the institution . We can assume the profit brought about by evaluation to the brand is $c(c>0)$, the input at the request of enterprise is $a(a>0)$, the further consultation costs of the enterprise is $e(e>0)$, the input of evaluation conducted by the consulting agency under the model of request is $b(b>0)$, the input under the independent evaluation model is $d(d>0)$ and the objective probability of the evaluation effect is $p(1 \geq p \geq 0)$. According to the objective fact, we should assume that the profit of the consulting agency is larger than 0 all the time, or $p e>b$ and $p e>d$. Also, we should assume the probabilities of the enterprise's adoption of "participate" strategy and of "not participate" strategy are respectively $\tilde{x}$ and $1-\tilde{x}$ respectively. The probabilities of evaluation at the request of enterprise and of independent evaluation are respectively $\tilde{y}$ and $1-\tilde{y}$. What is worth noticing is that an enterprise has an absolute right of autonomy in evaluation. According to 
the strategy set of game participants, the following matrix may be obtained as shown in Table 2 .

Table 2. Profit matrix of participating enterprise and evaluating institution evaluating institution

\begin{tabular}{|l|c|c|}
\hline & Request $(\tilde{y})$ & Independent $(1-\tilde{y})$ \\
\hline Participate $(\tilde{x})$ & $(-a-e, p c-a, c-a),(-b, p e-b, e-b)$ & $(0, p c-e, c),(d, p e-d, e-d)$ \\
\hline Not P. $(1-\tilde{x})$ & $(0,0,0),(-b, 0,0)$ & $(0, p c, c),(-d, 0,0)$ \\
\hline
\end{tabular}

We calculate the follows according to Eqs. (2) and (3):

$V\left(\tilde{a}_{11} \geq \tilde{a}_{i 1}, i=1,2\right)=\min \left\{V\left(\tilde{a}_{11} \geq \tilde{a}_{11}\right), V\left(\tilde{a}_{11} \geq \tilde{a}_{21}\right)\right\}=\min \left\{1, V\left(\tilde{a}_{11} \geq \tilde{a}_{21}\right)\right\}$, $V\left(\tilde{b}_{11} \geq \tilde{b}_{i 1}, i=1,2\right)=\min \left\{V\left(\tilde{b}_{11} \geq \tilde{b}_{11}\right), V\left(\tilde{b}_{11} \geq \tilde{b}_{21}\right)\right\}=1$.

Similarly, we may calculate as follows:

$V\left(\tilde{a}_{12} \geq \tilde{a}_{i 2}, i=1,2\right)=1$,

$V\left(\tilde{a}_{21} \geq \tilde{a}_{i 1}, i=1,2\right)=\min \left\{1, V\left(\tilde{a}_{21} \geq \tilde{a}_{11}\right)\right\}$,

$V\left(\tilde{a}_{22} \geq \tilde{a}_{i 2}, i=1,2\right)=1$,

$V\left(\tilde{b}_{12} \geq \tilde{b}_{i 2}, i=1,2\right)=1$,

$V\left(\tilde{b}_{21} \geq \tilde{b}_{i 1}, i=1,2\right)=\frac{b}{p e+b}$,

$V\left(\tilde{b}_{22} \geq \tilde{b}_{i 2}, i=1,2\right)=1$.

Then:

(1) When $p c>a$ or the profit brought about by evaluation under the model of request is larger than the input requested by the enterprise:

$V\left(\tilde{a}_{11} \geq \tilde{a}_{i 1}, i=1,2\right)=1$,

$V\left(\tilde{a}_{12} \geq \tilde{a}_{i 2}, i=1,2\right)=\frac{c}{e+c}$,

$V\left(\tilde{a}_{21} \geq \tilde{a}_{i 1}, i=1,2\right)=\min \left\{1, \frac{a+e}{p c-a}\right\}$.

a) If the profit of the enterprise when participating in brand evaluation is lower than the costs of the enterprise's participation in evaluation and further consultation or $p c-a>a+e$, then:

$V\left(\tilde{a}_{21} \geq \tilde{a}_{i 1}, i=1,2\right)=\frac{a+e}{p c-a}$.

We may get the follows by further reference to Eq. (5):

$V_{11}=V\left(\tilde{a}_{11} \geq \tilde{a}_{i 1}, i=1,2\right) \cdot V\left(\tilde{b}_{11} \geq \tilde{b}_{i 1}, i=1,2\right)=1$.

Similarly:

$V_{12}=\frac{c}{a+c}, \quad V_{21}=\frac{(a+e) \cdot b}{(p c-a) \cdot(1+p) e}, \quad V_{22}=1$

According to Definition 4, \{participate, independent\}, \{not participate, request\} and \{not participate, independent $\}$ are the optimal pure strategy solutions when the possibility degree is 1 , $c /(a+c)$ and $((a+e) \cdot b) /((p c-a) \cdot(1+p) e)$.

b) If the profit of the enterprise when participating in brand evaluation is lower than the costs of the enterprise's participation in evaluation and further consultation or $p c-a=a+e$, then: 
$V\left(\tilde{a}_{21} \geq \tilde{a}_{i 1}, i=1,2\right)=1$.

\{Participate, independent\}, \{not participate, request $\}$ and not participate, independent $\}$ are the optimal pure strategy solution to $c /(a+c), b /((1+p) e)$ and 1 when the possibility degree of the game is 1 .

(2) When $p c<a$, the profit brought about by evaluation under the request model is smaller than the input requested by the enterprise, then \{Participate, independent\}, \{not participate, request and not participate, independent\} are the optimal pure strategy solutions when the possibility degree is $c /(a+c), 1,1$.

\section{Case application and analysis}

Example 1: The triangular fuzzy profit matrix of the participating enterprise and the evaluating institution is shown in Table 3 below. The profit brought about by evaluation to the brand is 300,000 Yuan; the input requested by the enterprise is 50,000 Yuan; the further consultation costs of the enterprise is 100,000 Yuan; the input of evaluation conducted by the consulting agency is 20,000 Yuan; the input under the independent evaluation model is 40,000 and the objective probability of the evaluation effect is 0.75 .

Table 3. Profit matrix case 1 of participating enterprise and evaluating institution (Unit: 10,000 Yuan)

\begin{tabular}{|c|c|c|}
\hline & Request $(\tilde{y})$ & Independent $(1-\tilde{y})$ \\
\hline Participate $(\tilde{x})$ & $(-15,17.5,25),(-2,5.5,8)$ & $(0,12.5,30),(4,3.5,6)$ \\
\hline Not P. $(1-\tilde{x})$ & $(0,0,0),(-2,0,0)$ & $(0,22.5,30),(-4,0,0)$ \\
\hline
\end{tabular}

So, $V_{11}=1, V_{12}=0.75, V_{21}=0$, and $V_{22}=0$. Thus, \{participate, request , \{not participate, independent $\}$ are the optimal pure strategy solutions when the possibility degree of the game is 1 , 0.75 .

Example 2: The profit brought about by evaluation to the brand is 150,000 Yuan; the input requested by the enterprise is 50,000 Yuan; the further consultation costs of the enterprise is 100,000 Yuan; the input of evaluation conducted by the consulting agency is 20,000 Yuan; the input under the independent evaluation model is 40,000 Yuan and the objective probability of the evaluation effect is 0.6 .

There is no way to get the optional strategy solution according to Definition 4 . According to Eq. (6) to (7), the optimal mixed strategy is $\{(0,0,1 / 3),(0,3 / 4,1)\}$, that is, the enterprise selects the "participate" strategy using fuzzy probability $(0,0,1 / 3)$; the "not participate" strategy using fuzzy probability $(2 / 3,1,1)$; the "request" strategy using fuzzy probability $(0,3 / 4,1)$ and independent strategy using fuzzy strategy $(0,1 / 4,1)$.

\section{Conclusions}

In this paper, the game problem in brand valuation where profits and losses are given in the form of triangular fuzzy number was studied. The fuzzy matrix game theory was expanded to the brand evaluation field. The methods for pure strategy judgment and mixed strategy solutions based on the brand valuation models for triangular fuzzy matrix game was proposed. The certain number is replaced by the fuzzy number so as to make the game match the practical evaluation better. However, in the triangular fuzzy matrix game of brand valuation, no game participant especially the enterprise's risk preference was taken into account. For the policy makers with different risk preferences, it is more likely for them to adopt different decisions. It is the issue to be further studied.

\section{Acknowledgements}

This research was financially supported by China Central Public-interest Scientific Institution 
Basal Research Fund (Grant Nos. 552016Y-4665 and 552016Y-4667 and 552015Y-3990) and National Key Technology Support Program (Grant Nos. 2015BAK46B02 and 2015BAK46B03-3).

\section{References}

[1] Zhang W. Game and Information Economics. Shanghai People's Publishing House, Shanghai, 1996.

[2] Chandra S., Aggarwal A. On solving matrix games with pay-offs of triangular fuzzy numbers: Certain observations and generalizations. European Journal of Operational Research, Vol. 246, 2015, p. 575-581.

[3] Li D. A fast approach to compute fuzzy values of matrix games with payoffs of triangular fuzzy numbers. European Journal of Operational Research, Vol. 223, 2012, p. 421-429.

[4] Adrian I., Coroianu L. Existence, uniqueness, calculus and properties of triangular approximations of fuzzy numbers under a general condition. International Journal of Approximate Reasoning, Vol. 62, 2015, p. 1-26.

[5] Rahaman S., Kumar N., Pal Madhumangal Solving Bi-matrix games with pay-offs of triangular intuitionistic fuzzy numbers. European Journal of Pure and Applied, Vol. 83, 2015, p. 184-192.

[6] Jiang Y., Fan Z. A practical method for priority of interval number complementary judgment matrix. System Engineering, Vol. 20, 2011, p. 89-92.

[7] Feng Z., Liu S. Consistency and priority of triangular fuzzy number complementary judgment matrix. Journal of Control and Decision, Vol. 21, 2006, p. 903-907.

[8] Wang Y., Jin J., Li M. Analysis of urban rail transit fire based on triangular fuzzy causality diagram. China Safety Science Journal (CSSI), Vol. 23, 2013, p. 22-26.

[9] Wang J., Chen W., Zhou Y., et al. Study of improved prioritizing algorithm based on triangular fuzzy number reciprocal judgment matrix. Computer Engineering and Applications, Vol. 50, 2014, p. 214-217.

[10] Wang T., Brazil T. Volterra mapping based behavioral modeling of nonlinear circuits and systems for high frequencies. IEEE Transactions on Microwave Theory and Techniques, Vol. 51, 2012, p. $1433-1440$. 